\title{
Capacidade do sistema de saúde nos municípios do Rio de Janeiro: infraestrutura para enfrentar a COVID-19
}

\author{
Giuliano Alves Borges e Silva 1 \\ Ernani Viana Saraiva 1 \\ Guilherme José Santos Nunes Ferreira 1 \\ Rogério de Mesquita Peixoto Junior ${ }^{1}$ \\ Luiz Felipe Ferreira ${ }^{1}$
}

1 Universidade Federal Fluminense / Instituto de Ciências da Sociedade de Macaé, Macaé / RJ - Brasil

Considerando um número crescente de casos de atendimento de emergência, provenientes do SARS-CoV-2, no Estado do Rio de Janeiro, o presente estudo se concentra no mapeamento da infraestrutura de saúde nos municípios do Estado, comparando o Índice de Eficiência em Estrutura antes (2016) e após a pandemia da COVID-19. O artigo preenche uma lacuna acadêmica ao informar aos especialistas, técnicos, formuladores e tomadores de decisão de políticas públicas de saúde, sobre a capacidade de cada localidade para enfrentar o problema. Para isso, calculamos o Índice de Eficiência da Estrutura (IEE), alocando os 92 municípios do Estado na curva de pandemia. Foi possível verificar que o Governo do Estado do Rio de Janeiro precisa começar a atuar na supressão da COVID-19, mantendo a política de abertura, ou reabertura, de leitos e aquisição de equipamentos. No entanto, também é necessário considerar as particularidades e deficiências de cada região, pois a política de transferência dos acamados para outras regiões com leitos disponíveis pode espalhar a doença.

Palavras-chave: COVID-19; curva de pandemia; capacidade do sistema de saúde; Índice de Eficiência em Estrutura (IEE); cidades do estado do Rio de Janeiro.

\section{Capacidad del sistema de salud en los municipios del estado de Río de Janeiro: infraestructura para enfrentar la COVID-19}

Considerando el número creciente de casos de atención de emergencia procedentes del SARS-CoV-2, en el estado de Río de Janeiro, este estudio se centra en el mapeo de la infraestructura de salud en los municipios de dicho estado, con el fin de informar a los especialistas, técnicos, formuladores y tomadores de decisiones de políticas de salud pública sobre la capacidad de cada localidad para enfrentar el problema. Para esto, calculamos el índice de eficiencia de estructura (IEE) colocando los 92 municipios del estado en la curva de la pandemia. Se pudo verificar que el Gobierno del Estado de Río de Janeiro debe comenzar a actuar para suprimir la COVID-19 manteniendo la política de apertura o reapertura de camas y adquisición de equipos. Sin embargo, también se deben considerar las particularidades y deficiencias de cada región, ya que la política de traslado de pacientes postrados a otras regiones con camas disponibles puede propagar la enfermedad.

Palabras clave: COVID-19; curva pandémica; capacidad del sistema de salud; índice de eficiencia de estructura (IEE); ciudades del estado de Río de Janeiro.

\section{Healthcare system capacity of the municipalities in the State of Rio de Janeiro: infrastructure to confront COVID-19}

Considering the growing number of cases requiring emergency care as a result of SARS-CoV-2 in the Brazilian State of Rio de Janeiro, this study focuses on mapping the health infrastructure of the municipalities of the state, comparing the Structure Efficiency Index (IEE) before (2016) and after the COVID-19 pandemic. The article fills a gap in the academic literature, informing public health policies specialists and technicians, as well as policy and decision-makers, about the capacity of municipalities to face the problem. We calculated the Structure Efficiency Index (IEE) of the states' 92 municipalities and positioned them on the pandemic curve. It was possible to verify that the government of the State of Rio de Janeiro needs to start acting to suppress COVID-19, maintaining the policy of providing more hospital beds, and purchasing equipment. However, it is also necessary to consider the particularities and deficiencies of each region, as the policy to transfer patients to places with available beds can contribute to the spread of the disease. Keywords: COVID-19; pandemic curve; healthcare system capacity; structure efficiency index (IEE); municipalities of the State of Rio de Janeiro. 


\section{INTRODUÇÃO}

Na luta contra a COVID-19, mesmo países com um alto número absoluto e relativo de leitos hospitalares de Unidade de Terapia Intensiva (UTI) exigiram leitos adicionais para atender ao pico de demanda por hospitalização (Baldwin \& Di-Mauro, 2020). Do ponto de vista sistêmico, essa circunstância é ampliada quando se observa um sistema de saúde já esgotado pela demanda usual, que pode prejudicar indiretamente pacientes de outras doenças e outras formas de tratamento em andamento.

Especificamente, no Brasil, menos de 10\% dos municípios possuem leitos de UTI, além de desigualdades nacionais internas, o que agrava o problema em regiões remotas (CNES, 2020). A quantificação da infraestrutura de saúde é relevante no combate ao SARS-CoV-2, pois auxilia na decisão sobre a política de isolamento social e na escolha de atividades econômicas prioritárias.

A capacidade de um sistema de saúde de lidar com a taxa de contaminação por SARS-CoV-2 no curto prazo é limitada (veja a linha representativa na Figura 1). Os recursos necessários são finitos, como leitos hospitalares, leitos de UTI, número de profissionais de saúde qualificados, ventiladores, respiradores, entre outros. Essa situação impõe um limite para o número de pacientes que podem ser tratados. A taxa de transmissão do vírus corona sobrecarregaria rapidamente qualquer sistema de saúde, deixando muitos pacientes infectados com condições pulmonares deterioradas sem nenhum tratamento, daí a necessidade de achatar a curva contaminada, impondo uma política drástica de distanciamento social (Gourinchas, 2020).

\section{FIGURA 1 CURVA DE PANDEMIA}

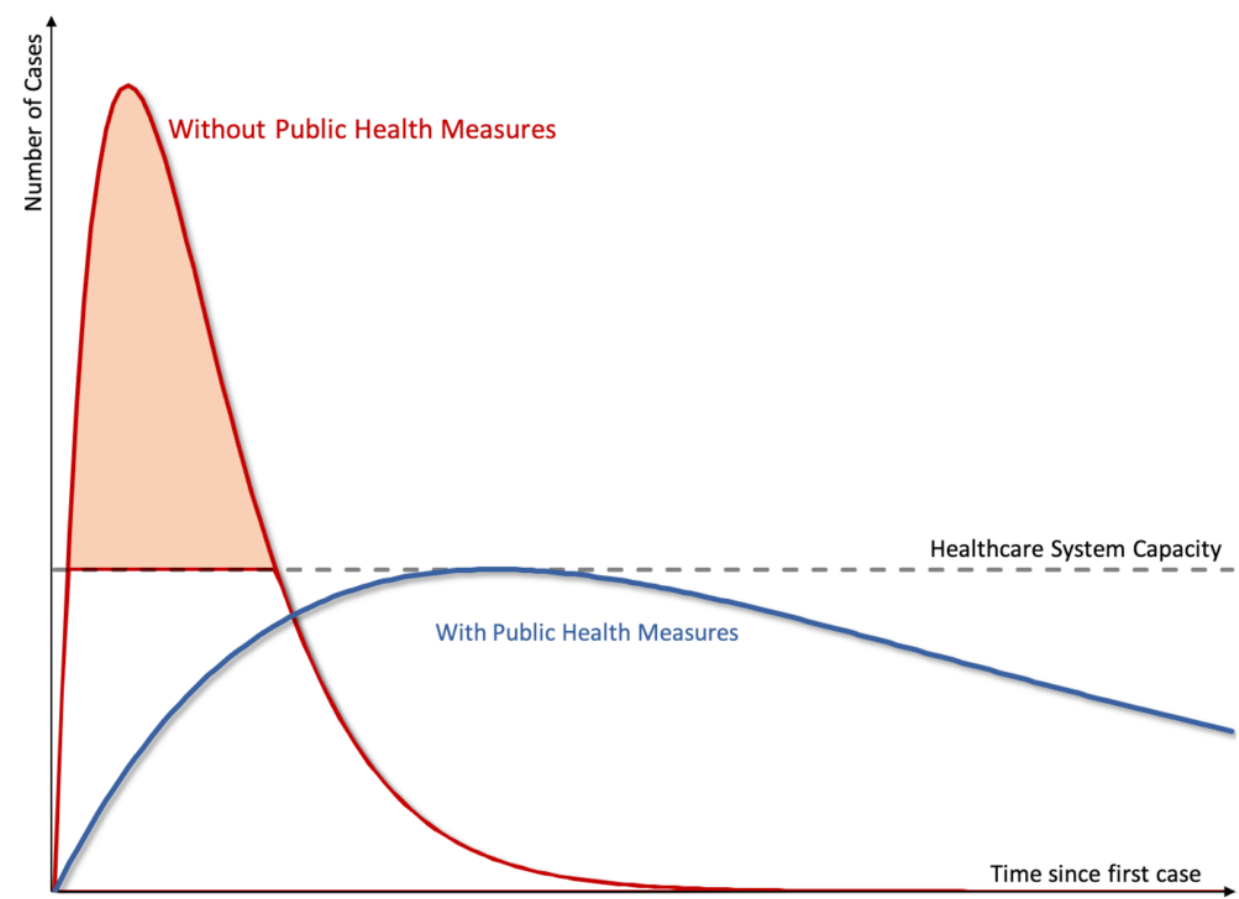

Fonte: Gourinchas (2020). 
Grande parte da estrutura necessária é apresentada pelo Cadastro Nacional de Estabelecimentos de Saúde (CNES). Nesta área, Mendes (2005) desenvolveu um método para calcular o Índice de Eficiência Técnica em Saúde (IETS), com base no Índice de Eficiência de Resultados (IER) e no Índice de Eficiência de Estrutura (IEE), este último com potencial para medir a capacidade de sistema de saúde em uma perspectiva comparativa. Por sua vez, a capacidade do sistema de saúde precisa ser definida para que políticas apropriadas sejam estabelecidas.

Nesse sentido, o presente estudo se concentra no mapeamento da infraestrutura de saúde nos municípios do Estado do Rio de Janeiro, comparando o Índice de Eficiência da Estrutura (IEE) antes (2016) e após a pandemia da COVID-19. O artigo preenche uma lacuna acadêmica, porque informa especialistas, técnicos, formuladores e tomadores de decisão de políticas públicas de saúde sobre a capacidade de cada localidade para enfrentar o problema. De uma perspectiva comparativa, localizará cada cidade do Estado na curva de achatamento pandêmica, de acordo com as condições de saúde.

Esta pesquisa é justificada por causa da recente declaração de uma pandemia da COVID-19 pela Organização Mundial da Saúde (OMS) em 11 de março, com um número crescente de casos de atendimento de emergência, vindos do SARS-CoV-2, no Estado de Rio de Janeiro. Considerando que a infraestrutura de saúde define a necessidade urgente de achatar a curva ascendente no número de casos, os pesquisadores pretendem fornecer dados e informações para a sociedade.

Consolidamos os dados coletados, a fim de apoiar os tomadores de decisão e a formulação de políticas públicas a entender os riscos de um possível relaxamento das medidas de isolamento social e quarentena anunciadas frente à crise econômica, com impacto em todos os estados e municípios.

\section{SÍNTESE TEÓRICA}

Há uma concordância de que as instituições governamentais devem desempenhar algum papel na área da saúde pública. É essencial observar que a situação do sistema de saúde se deve a uma combinação de mercado e governo e que a eficiência gerada nesse segmento dependerá de como é organizada financeiramente. Por sua vez, não é possível afirmar que existe um modelo de organização financeira a ser considerado ideal, equilibrando os investimentos públicos e privados em saúde. Consequentemente, podemos inferir que o sistema de saúde privado trará maior eficiência, enquanto o sistema público de saúde seria eficaz, ainda que apenas em bens e serviços essenciais de saúde. O Sistema Único de Saúde (SUS) é considerado uma proposta generosa e universal, embora enfrente problemas de fornecimento precário de bens e serviços em algumas comunidades (Farias \& Melamed, 2003).

Há uma discussão sobre os impactos positivos da política de saúde pública no Brasil, baseada principalmente na iniciativa de fornecer medicamentos gratuitos e sua forma universal de distribuição por meio de farmácias populares e básicas. Também é necessário considerar toda a multiplicidade demográfica da população brasileira e os padrões de qualidade de saúde das distintas regiões do país. Mesmo assim, pode-se afirmar que o SUS e sua expansão reduziram com sucesso o número de doenças na população, superando as desigualdades no Brasil (Souza et al., 2018).

Os princípios norteadores do SUS, e toda a sua base legal de apoio, devem causar mudanças profundas, como observado na redução da taxa de mortalidade e no aumento da expectativa de vida da população. No entanto, ainda é necessário melhorar o sistema governamental em uma ação coerente e articulada à proteção social (Souza et al., 2018).

A implantação do SUS é considerada a maior política social criada no país, apesar de conter tantas dificuldades e problemas que não foram superados nas suas três décadas de existência. Esses problemas 
persistem porque nunca houve um partido político ou governo que assumiu a responsabilidade e resolveu adequadamente os problemas mencionados e colocou o SUS em seu devido lugar de destaque (Rizzotto, 2018).

No entanto, em tempos de doenças ocasionadas pelo vírus Corona (COVID-19), é crucial reavaliar a infraestrutura de saúde do país seguindo as orientações da Organização Mundial da Saúde (OMS), como o Regulamento Internacional de Saúde da OMS (WHO IHR MEF), que visa fornecer uma visão geral precisa de cada país. Dessa maneira, é possível verificar as capacidades para garantir níveis adequados de saúde pública. Esse monitoramento é realizado com um conjunto de componentes: (1) um relatório obrigatório de capacidade - o Relatório Anual de Auto-Avaliação do Estado - SPAR), (2) a Avaliação Externa Conjunta, (3) As Revisões Pós-Ações e (4) os Exercícios de Simulação. É necessário dizer que esta medida não leva em consideração outros componentes indiretos críticos "que podem comprometer o controle de epidemias emergentes, como condições demográficas, ambientais, socioeconômicas e políticas" (Gilbert et al., 2020, p. 873).

A COVID-19 criou uma crise no Sistema de Saúde em todo o mundo, o que levou todos os setores de saúde governamentais e privados a desenvolver várias estratégias para absorver principalmente a expansão acelerada da demanda de UTI. Essa demanda de UTI é particularmente assustadora para o público em geral, uma vez que a UTI é percebida como um local onde as pessoas vão quando estão morrendo, carregando dessa maneira estresse físico e mental, não apenas para o paciente, mas também para sua família (Paneru, 2020).

Keesara, Jonas, e Schulman (2020) sugerem a observação de três áreas: (1) avaliação do atendimento clínico usando (2) reembolso por novos serviços tecnológicos e (3) um alívio regulatório ampliado. Eles também consideram que os modelos hospitalares em casa seriam uma alternativa para pacientes infectados, mas estáveis, ou uma alta mais rápida dos pacientes admitidos na COVID-19.

Maves et al. (2020) declaram a importância de um sistema de triagem eficiente para alocar todos os recursos de cuidados críticos disponíveis, uma vez que as demandas clínicas no surto excederão amplamente a capacidade provisionada de recursos essenciais. Eles argumentam que um protocolo de suporte à decisão de triagem entre infraestruturas e treinamento pessoal é necessário ser estabelecido, observando a atenção às proteções legais e regulamentares. Esses fatores devem ser adequados a três níveis diferentes de capacidade que podem ocorrer devido à progressão do pico: a capacidade convencional, a contingencial e a capacidade na fase crítica. Na capacidade convencional, um hospital deve ser capaz de expandir sua capacidade de cuidados intensivos. Na capacidade de contingência que ocorre durante um desastre, é necessário expandir a capacidade de cuidados críticos em 100\%. Se a situação se desenvolver a um nível catastrófico, que é a tendência por causa da COVID-19, haverá severas restrições de recursos, e o hospital e todo o público principalmente afetado devem considerar um protocolo de triagem.

Outra estratégia essencial para evitar uma crise de infraestrutura é o isolamento domiciliar em vez do isolamento hospitalar, principalmente orientado a pacientes com sintomas leves a moderados da COVID-19. No entanto, vários formuladores de políticas como os chineses, por exemplo, são contra essa estratégia porque coloca os familiares do paciente em risco, o que também afeta psicologicamente o paciente (Chen et al., 2020). Mesmo assim, é necessário aplicar medidas estritas de detecção, controle e prevenção, incluindo "vigilância reforçada e identificação rápida de casos suspeitos, seguidas de transferência e isolamento do paciente, seguidas de diagnóstico rápido, rastreamento e acompanhamento de contatos em potencial" (Gilbert et al., 2020, p. 871). 
Um excelente exemplo de rápida adaptação da infraestrutura ao surto da COVID-19 são os modelos de hospitais Fangcang implementados na China. A expressão Fangcang simbolizava a metáfora da Arca de Noé e era usada principalmente em hospitais militares de campo que convertem grandes construções, como estádios, por exemplo, em hospitais temporários para atender emergências de saúde pública. Esses hospitais abrigos têm três características principais para lidar com uma crise como o surto da COVID-19: (1) construção rápida, pois apenas adapta outras grandes estruturas físicas; (2) escala maciça do sistema de saúde, uma vez que aumenta muito rapidamente a capacidade de assistência à saúde e (3) o baixo custo de operação dessas instalações (Chen et al., 2020).

Um exemplo altamente ineficiente de infraestrutura é observado no Nepal, onde, além dos esforços para expandir seus leitos sobrecarregados de UTI, não há profissionais de saúde suficientes treinados para administrar o nível III de UTI exigido por pacientes COVID-19 em estado crítico. A UTI Nível III I, para pacientes que necessitam de suporte de dois ou mais órgãos ou ventilação mecânica sozinha, é composta por uma enfermeira por paciente idealmente e um médico presente 24 horas. Até o maior hospital universitário do Nepal possui menos do que o mínimo recomendado de 5\% dos leitos de UTI do total de leitos disponíveis. Além disso, em todo o país, existem 480 leitos de UTI e 260 ventiladores nos setores público e privado (Paneru, 2020).

Pelo menos, a OMS está apoiando os países africanos para melhorar sua capacidade de diagnóstico, uma vez que costumavam ter apenas dois laboratórios de referência para atender a quase todos os países de lá. O principal desafio é fornecer treinamento rápido para capacitar o pessoal para executar os testes e assegurar uma estocagem correta de materiais envolvidos nos testes. Esse treinamento é crucial para reduzir o tempo de entrega de resultados, essencial para o planejamento de políticas de saúde (Gilbert et al., 2020).

\section{METODOLOGIA}

Para este artigo, o IEE será considerado como a "capacidade do sistema de saúde", pois abrange:

- Gastos municipais per capita SUS - impacta nas condições de investimento da cidade em demandas de saúde e emergência, compra de respiradores, disponibilidade de novos leitos, entre outros (2016 e 20201);

- Transferências per capita da União para o SUS - impacta nas condições de investimento da cidade em demandas de saúde e emergência, compra de respiradores, disponibilidade de novos leitos, entre outros (2016 e 2020);

- Leitos hospitalares por habitante - afeta diretamente a capacidade de serviço (2016 e 2020);

- Médicos por mil habitantes - influencia nas condições de atendimento direto (2016 e 2020);

- Outros profissionais de saúde por mil habitantes - influencia nas condições de atendimento direto (2016 e 2020);

- Equipes de Saúde da Família - influencia na realocação das atividades de saúde, essencial para a manutenção do sistema de saúde (2016 e 2020).

${ }^{1}$ O número de habitantes em 2020 é calculado com base nos dados de 2019. 
As curvas de achatamento do número de casos, comparadas à capacidade dos sistemas de saúde, consideram o padrão de dispersão dos países ricos, atribuindo uma média de 0,5 à capacidade de atendimento do sistema de saúde, como um indicador intermediário (Walker et al., 2020). Nos países em desenvolvimento, os resultados da pandemia podem ser piores do que o previsto, dadas as condições de saneamento, o abastecimento de água e as aglomerações de pessoas nas comunidades (Walker et al., 2020). O cálculo do IEE considera a média de seis indicadores projetados, para alocar valores entre os próprios municípios, onde o pior município do Estado em cada indicador possui pontuação zero no respectivo item. Por outro lado, o município melhor recebe uma pontuação de 1 . O IEE é calculado usando a seguinte fórmula:

$$
I E E=1-\left(\frac{R \text { melhor }-R \text { calc }}{R \text { melhor }-R \text { pior }}\right)
$$

Onde: "R melhor" representa o melhor resultado para cada variável (como, por exemplo, Camas Hospitalares por habitante ou Profissionais de saúde por habitante). "R calc" corresponde ao indicador de município a ser calculado na variável e "R pior" refere-se ao pior resultado para cada variável (Mendes, 2005).

As informações disponíveis para todos esses indicadores no banco de dados CNES (fonte de dados secundários) de todos os municípios do Estado do Rio de Janeiro são de 2016, o que corresponde a uma limitação da pesquisa. No entanto, algumas modificações foram observadas no cenário. Diante da pandemia do vírus corona, os municípios tiveram que atualizar os dados da estrutura de saúde, desde abril de 2020, devido à rápida expansão do número de leitos e equipamentos hospitalares. Essa circunstância possibilitou a comparação do IEE em maio de 2016 com o atual IEE (maio de 2020), permitindo avaliar quais municípios do estado melhoraram ou regrediram suas respectivas infraestruturas em perspectiva comparativa. Todos os noventa e dois municípios do Estado do Rio de Janeiro participaram da pesquisa, e o estudo apresenta os resultados do IEE em cada município. A análise e a interpretação dos dados usam um gráfico explicativo com base nas condições dos serviços para uma demonstração visual. Em seguida, os pesquisadores apresentam suas análises, conclusões e sugestões ao Governo do Estado e, consequentemente, a todos os Municípios. As estimativas de óbitos e necessidades de cuidados foram baseadas em Walker et al. (2020).

\section{RESULTADOS E DISCUSSÃO}

\subsection{Resultados de 2016}

Trinta e cinco municípios do Estado (38\%) - com IEE entre 0,0 e 0,2 - apresentavam condições deploráveis de infraestrutura de saúde para lidar com casos da COVID-19. Nesses locais, com a estrutura anterior, os casos precisariam ser tratados em outros municípios, ou os leitos de emergência deveriam ser disponibilizados com urgência dentro de um mês após o primeiro caso confirmado (ver Figura 2 e Figura 3). 


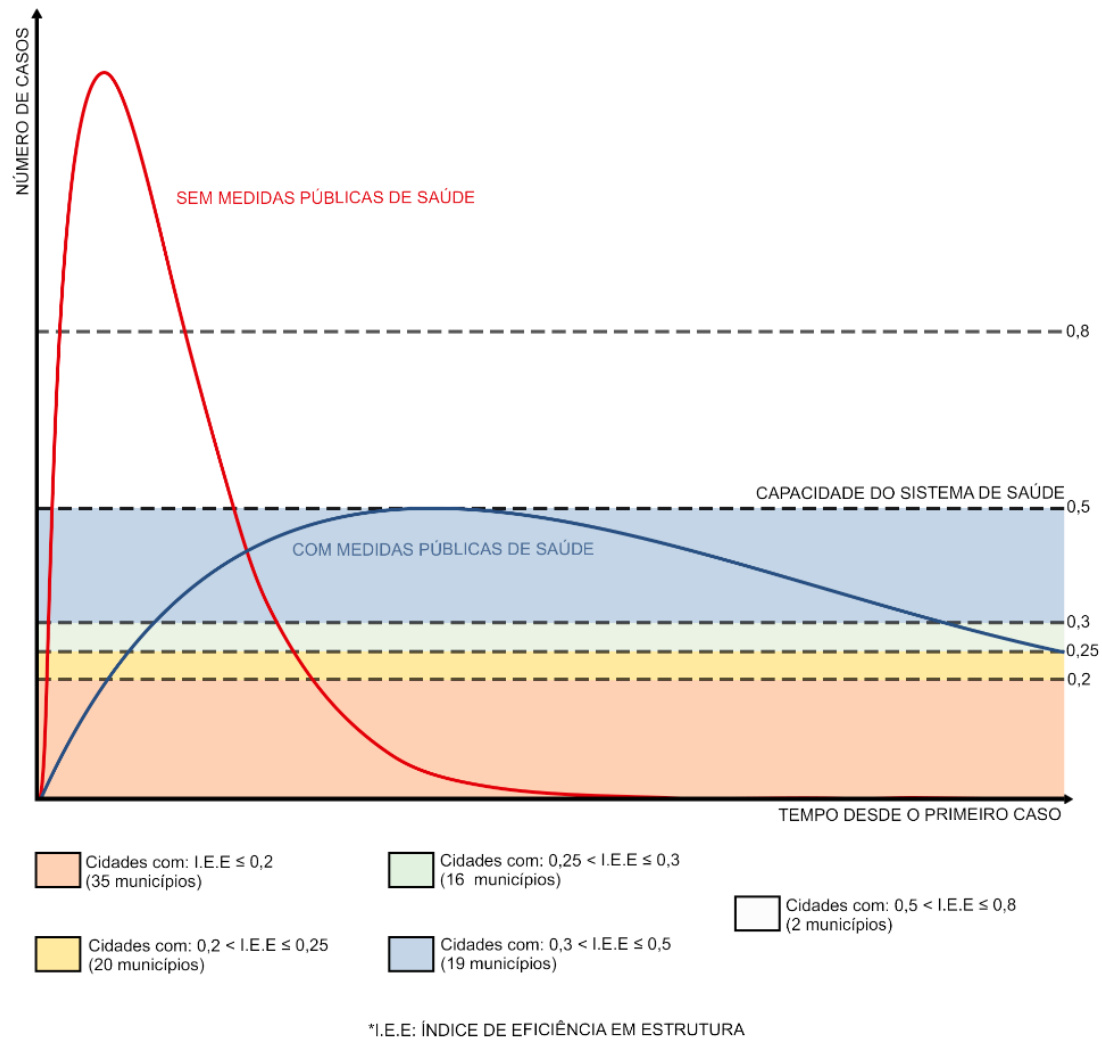

Fonte: Baseada em Gourinchas (2020).

Complementando esse número alarmante, o estado possuía 20 municípios com IEE entre 0,2 e 0,25 e más condições de atendimento, além de outros 16 municípios entre 0,25 e 0,3, ainda insatisfatórios, mesmo com o achatamento da curva no número de casos.

Em condições normais, apenas 21 municípios possuíam capacidade inicial de atendimento (considerando 3 meses após o primeiro caso) para os casos da COVID-19, onde Niterói $(0,51)$ e Vassouras $(0,766)$ seriam os únicos capazes de atender à demanda da cidade dentro da curva achatada, sem considerar os serviços de emergência dos municípios vizinhos. A regionalização dos leitos de emergência precisaria ser intensificada de acordo com o estudo das condições locais, devido à estratificação dos dados.

Dito isto, é necessário discutir que mesmo a desaceleração atual da curva em direção ao achatamento não foi capaz de mitigar os efeitos no sistema de saúde, de acordo com os critérios metodológicos. Por esse motivo, os municípios do Rio de Janeiro precisariam expandir sua capacidade de atendimento. Em abril de 2020, as capacidades já eram diferentes, conforme descreve o tópico 4.2 a seguir. 


\title{
FIGURA 3 IEE 2016 NOS MUNICÍPIOS DO RIO DE JANEIRO
}

\author{
*I.E.E: ÍNDICE DE EFICIÊNCIA EM ESTRUTURA
}

Cidades com: I.E.E $\leq 0,2$ - Japeri, São João de Meriti, Seropédica, Belford Roxo, Queimados, São Pedro da Aldeia, Magé, São Gonçalo, São Francisco de Itabapoana, Saquarema, São Fidélis, Maricá, Rio das Ostras, Mesquita, Sumidouro, Guapimirim, Conceição de Macabu, Varre-Sai, Bom Jardim, Teresópolis, Paty dos Alferes, Duas Barras. Nova Iguaçu, Araruama, Mendes, Paraty, São José do Vale do Rio Preto, Barra do Piraí, Cachoeiras de Macacu, Trajano de Moraes, Duque de Caxias, Italva, Miracema, Itaboraí, Tanguá.

Cidades com: 0,2 < I.E.E $\leq$ 0,25 - Santo Antônio de Pádua, Porcíuncula, Pinheiral, Itaocara, Iguaba Grande, Cordeiro, Paracambi, Nova Friburgo, Nilópolis, Cardoso Moreira, Cabo Frio, Cantagalo, Casemiro de Abreu, Areal, Aperibé, Arraial do Cabo, Santa Maria Madalena, Barra Mansa, Silva Jardim, Valença.

Cidades com: 0,25 < I.E.E $\leq$ 0,3 - Itaguaí, Rio Claro, Mangaratiba, Miguel Pereira, Engenheiro Paulo de Frontin, Rio das Flores, Bom Jesus de Itabapoana, São Sebastião do Alto, Volta Redonda, Itatiaia, Paraíba do Sul, Laje do Muriaé, Resende, Sapucaia, Cambuci, Macaé.

Cidades com: 0,3 < I.E.E $\leq$ 0,5 - Rio de Janeiro, Petrópolis, Rio Bonito, Armação dos Búzios, Campos dos Goytacazes, Angra dos Reis, Carmos, Natividade, Carapebus, Três Rios, Piraí, Comendador Levy Gasparian, São José de Ubá, Quissamã, Macuco, São João da Barra, Quatis, Porto Real, Itaperuna.

Cidades com: 0,5 $<$ I.E.E $\leq 0,8-$ Niterói, Vassouras.

Fonte: Elaborada pelos autores.

\subsection{Resultados em abril/2020}

Conforme as características típicas do IEE ao estabelecer um indicador "classificatório", foi possível observar poucas mudanças na distribuição quantitativa dos municípios entre os grupos. Face aos dados atuais, trinta e três cidades mostram infraestrutura de saúde deplorável (36\%) (o número era trinta e cinco em 2016). Percebemos que a respectiva classificação de algumas cidades piora e se junta a esse grupo - com IEE entre 0 e 0,2 - como no caso de Pinheiral, Iguaba Grande, Cordeiro, Itaocara, Porciúncula, Nilópolis, Paracambi, Santa Maria Madalena, Sapucaia, Itaguaí e Quatis (ver Figuras 4 e 5).

Os municípios que saíram da pior faixa de qualificação foram: Itaboraí, Trajano de Moraes, Tanguá, Barra do Piraí, Paty do Alferes, Paraty, Mendes, Teresópolis, Duas Barras, Bom Jardim, São Fidélis, Maricá e Sumidouro.

Dezenove municípios formam a segunda faixa com más condições de serviços de saúde, IEE entre 0,2 e 0,25, e outras vinte e quatro cidades entre 0,25 e 0,3, ainda em condições insatisfatórias. 


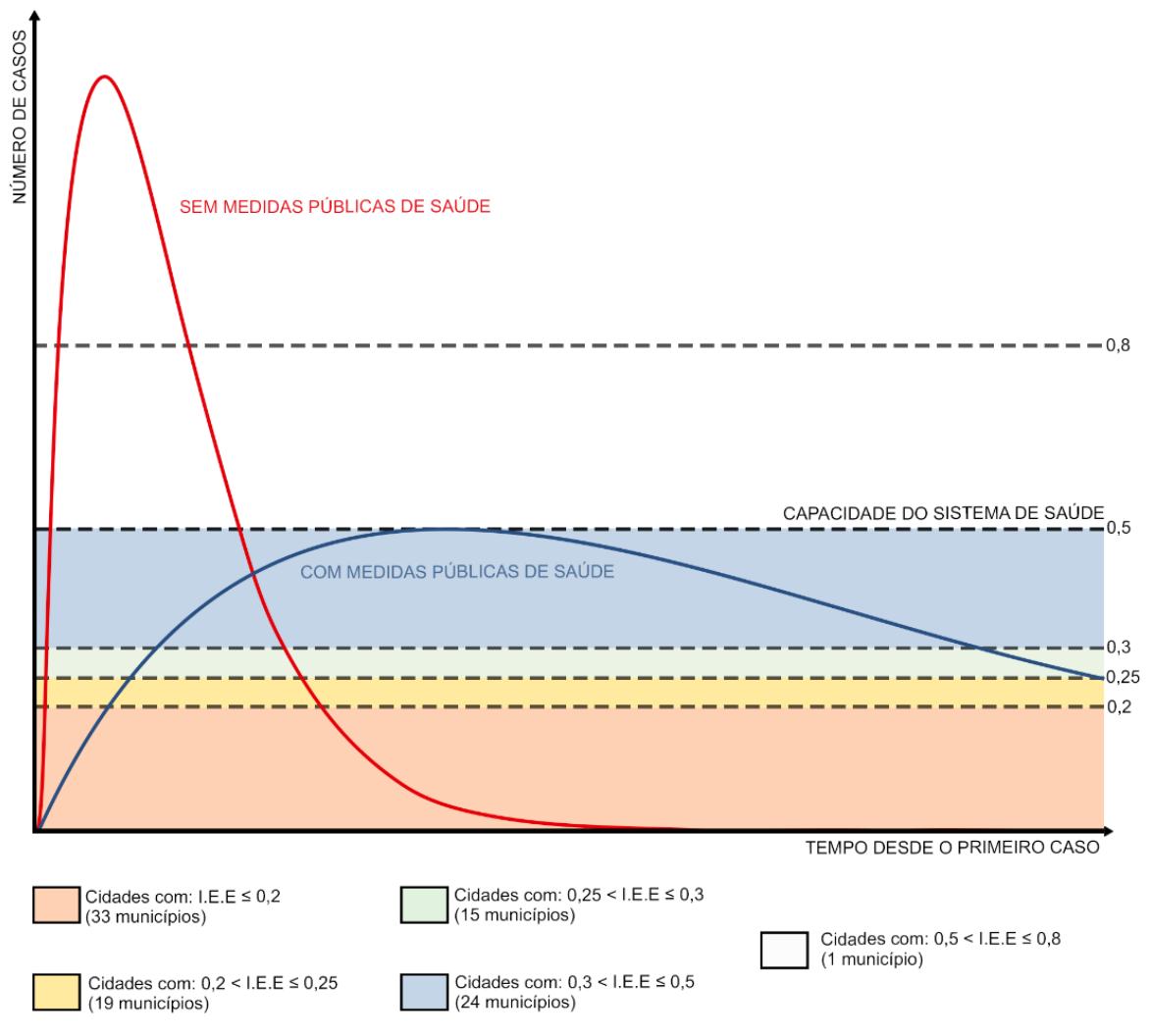

*I.E.E: INDICE DE EFICIÊNCIA EM ESTRUTURA

Fonte: Elaborada pelos autores.

\section{FIGURA 5 IEE 2020 NOS MUNICÍPIOS DO RIO DE JANEIRO}

\section{*I.E.E: ÍNDICE DE EFICIÊNCIA EM ESTRUTURA}

Cidades com: I.E.E $\leq$ 0,2 - Belford Roxo, Seropédica, São Pedro da Aldeia, Queimados, São João de Meriti, São Francisco de Itabapoana, Japeri, São Gonçalo, Magé, Saquarema, Conceição de Macabu, Itaguaí, Rio das Ostras, Mesquita, Cachoeiras de Macacu, Porciúncula, São José do Vale do Rio Preto, Quatis, Nova Iguaçu, Paracambi, Santa Maria Madalena, Nilópolis, Araruama, Itaocara, Cordeiro, Miracema, Varre-Sai, Sapucaia, Iguaba Grande, Duque de Caxias, Guapimirim, Pinheiral, Italva.

Cidades com: 0,2 < I.E.E $\leq 0,25$ - Trajano de Moraes, Paty do Alferes, Barra do Piraí, Arraial do Cabo, Engenheiro Paulo de Frontin, Itaboraí, São Fidélis, Paraty, Tanguá, Mendes, Santo Antônio de Pádua, Silva Jardim, Teresópolis, Casimiro de Abreu, Barra Mansa, Mangaratiba, Bom Jardim, Cabo Frio, Duas Barras.

Cidades com: 0,25 < I.E.E $\leq$ 0,3 - Itatiaia, São José de Ubá, Nova Friburgo, Rio Bonito, Maricá, Cantagalo, Piraí, Cambuci, Aperibé, Resende, Laje do Muriaé, Volta Redonda, Paraíba do Sul, Bom Jesus do Itabapoana, Rio de Janeiro.

Cidades com: 0,3 < I.E.E $\leq$ 0,5 - Rio das Flores, Valença, Areal, Carapebus, Três Rios, Sumidouro, Campos dos Goytacazes, Cardoso Moreira, Macaé, Itaperuna, Comendador Levy Gasparian, Natividade, Carmo, São João da Barra, Angra dos Reis, São Sebastião do Alto, Miguel Pereira, Armação dos Búzios, Petrópolis, Macuco, Rio Claro, Porto Real, Niterói, Quissamã.

Cidades com: $0,5<$ I.E.E $\leq 0,8$ - Vassouras.

Fonte: Elaborada pelos autores. 
Considerando os critérios arbitrados e fundamentados em condições internacionais, e levando em conta a evolução da estrutura pós COVID-19, 26 cidades seriam capazes de atender a população durante os três primeiros meses, uma vez que podem sustentar a curva epidêmica atenuada. Nesse sentido, o município de Vassouras é o destaque. Embora a cidade tenha uma população pequena, possui um importante hospital universitário, além de leitos específicos disponíveis para atender à demanda da COVID-19.

\subsection{Discussões e reflexões}

O IEE não pode ser considerado um instrumento para medir a estrutura de serviço para as complicações advindas especificamente da COVID-19. Porém, traz a capacidade de explicar a estrutura do sistema de saúde, pois reúne a capacidade de articulação da rede como um todo, equipe, possibilidade de aplicação de recursos e leitos. A introdução de um indicador com essas características se torna significativa porque o enfrentamento ao vírus corona minimiza a capacidade de serviço relacionada a outras doenças, criando um problema em todo o sistema.

Não se pode argumentar contra o recente incremento da infraestrutura em saúde, a partir da lógica de que a nova estrutura pode ficar ociosa logo após o término do surto do vírus corona. Pelo contrário, o sistema de saúde precisará suprir a demanda relacionada a outras doenças. Foi detectado, em muitos países, um aumento de distúrbios cardiovasculares e psiquiátricos, sem mencionar a situação de cirurgias eletivas temporariamente canceladas (Linschoten \& Asselbergs, 2020; Shigemura et al., 2020; Zheng, Boni, \& Fingerhut, 2020). Todas as circunstâncias listadas, se observadas em conjunto, indicam que, durante muito tempo, haverá uma maior demanda por serviços de saúde após o período de pandemia.

Como resultado do referido contexto, a política de abertura de leitos, iniciada pelo Governo do Estado no Hospital Zilda Arns, Hospital Anchieta e no Instituto do Cérebro, precisa ser intensificada, com a distribuição regional. Portanto, é necessário mapear a infraestrutura provisória, permanente ou não utilizada nos municípios. É possível formular uma ação de curto prazo com base no intercâmbio de informações. A estratificação dos dados do IEE pode contribuir para a formulação desta decisão.

É necessária uma mobilização da estrutura da saúde de maneira descentralizada, contemplando as desigualdades regionais. O procedimento de transferência de pacientes dentro das unidades hospitalares já é motivo de risco, consequentemente, ao considerar a transferência de pacientes entre as cidades, o risco é alto. Isso se explica porque o vírus tem um alto potencial de propagação, mesmo em hospitais e entre profissionais de saúde (Brindle \& Gawande, 2020). Se mal conduzida, uma política pública dessa natureza pode espalhar o surto para cidades menos afetadas.

Observando a evolução do IEE entre os anos de 2016 e 2020, ambas as cidades de Maricá e Sumidouro, subiram mais de uma posição no ranking. Também podem ser destacadas outras cidades que alavancaram seus índices em mais de 0,1: Rio Claro, Miguel Pereira, Cardoso Moreira e Quissamã. Enquanto que as cidades de Itaguaí, Itaperuna, São José de Ubá e Quatis apresentaram queda acima de 0,1 no IEE (Tabela 1 ). 
RAP | Capacidade do sistema de saúde nos municípios do Rio de Janeiro: infraestrutura para enfrentar a COVID-19

TABELA 1 EVOLUÇÃO DO IEE NAS CIDADES DE PIOR CLASSIFICAÇÃO

\begin{tabular}{|c|c|c|c|c|}
\hline \multirow[b]{2}{*}{ Grupo } & \multirow[b]{2}{*}{ Cidades } & \multicolumn{2}{|c|}{ I.E.E. } & \multirow[b]{2}{*}{ Acréscimo ou Decréscimo } \\
\hline & & 2016 & 2020 & \\
\hline \multirow{18}{*}{4} & Duas Barras & 0,161 & 0,248 & 0,087 \\
\hline & Cabo Frio & 0,224 & 0,248 & 0,024 \\
\hline & Bom Jardim & 0,150 & 0,242 & 0,093 \\
\hline & Mangaratiba & 0,254 & 0,242 & $-0,012$ \\
\hline & Barra Mansa & 0,242 & 0,241 & $-0,001$ \\
\hline & Casimiro de Abreu & 0,238 & 0,240 & 0,002 \\
\hline & Teresópolis & 0,158 & 0,233 & 0,075 \\
\hline & Silva Jardim & 0,244 & 0,233 & $-0,011$ \\
\hline & Santo Antônio de Pádua & 0,201 & 0,228 & 0,027 \\
\hline & Mendes & 0,169 & 0,226 & 0,057 \\
\hline & Tanguá & 0,199 & 0,226 & 0,028 \\
\hline & Paraty & 0,173 & 0,226 & 0,053 \\
\hline & São Fidélis & 0,111 & 0,213 & 0,102 \\
\hline & Itaboraí & 0,196 & 0,212 & 0,017 \\
\hline & Engenheiro Paulo de Frontin & 0,259 & 0,212 & $-0,046$ \\
\hline & Arraial do Cabo & 0,241 & 0,211 & $-0,029$ \\
\hline & Barra do Piraí & 0,181 & 0,211 & 0,030 \\
\hline & Paty do Alferes & 0,159 & 0,211 & 0,052 \\
\hline \multirow{13}{*}{5} & Trajano de Moraes & 0,190 & 0,210 & 0,020 \\
\hline & Italva & 0,194 & 0,198 & 0,004 \\
\hline & Pinheiral & 0,205 & 0,197 & $-0,008$ \\
\hline & Guapimirim & 0,134 & 0,197 & 0,063 \\
\hline & Duque de Caxias & 0,190 & 0,196 & 0,006 \\
\hline & Iguaba Grande & 0,210 & 0,194 & $-0,016$ \\
\hline & Sapucaia & 0,279 & 0,194 & $-0,085$ \\
\hline & Varre-Sai & 0,144 & 0,192 & 0,048 \\
\hline & Miracema & 0,195 & 0,192 & $-0,003$ \\
\hline & Cordeiro & 0,211 & 0,191 & $-0,019$ \\
\hline & Itaocara & 0,209 & 0,189 & $-0,020$ \\
\hline & Araruama & 0,167 & 0,181 & 0,014 \\
\hline & Nilópolis & 0,217 & 0,179 & $-0,039$ \\
\hline
\end{tabular}




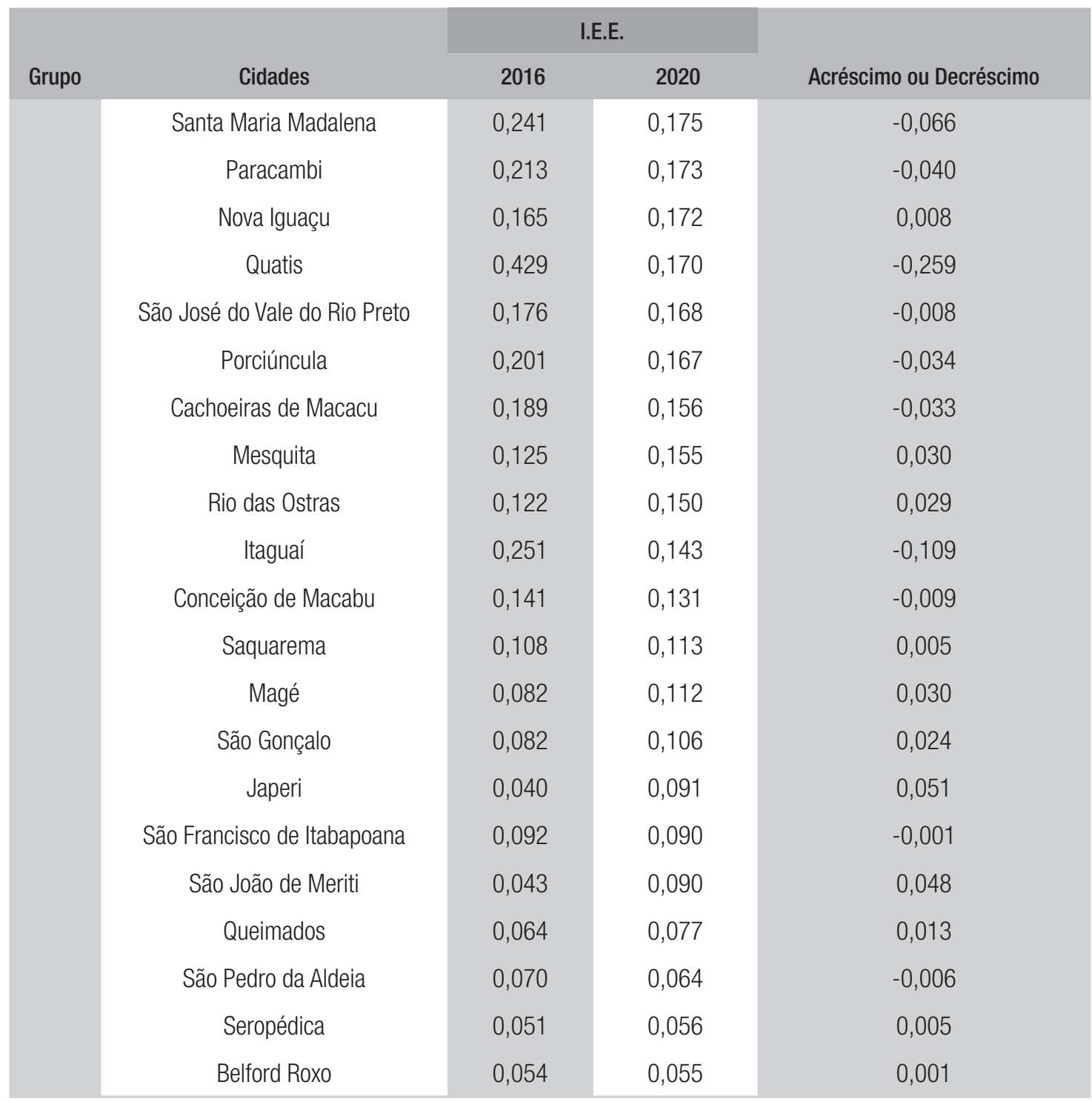

Fonte: Elaborada pelos autores.

Sobre o cálculo do IEE em todos os municípios fluminenses, quando é relatado um caso da COVID-19, recomenda-se que nenhuma localidade com um índice menor que 0,25 (Tabela 1) hipotetize um relaxamento das provisões de distanciamento social. Essa análise é capaz de auxiliar os formuladores de políticas em decisões semelhantes às estabelecidas pelo Decreto 47.025 (2020), autorizando prefeitos a liberar atividades comerciais nos municípios sem notificação.

Em termos absolutos, os indicadores de eficiência apontam Vassouras, Quissamã, Niterói, Porto Real e Rio Claro, como cidades com boa capacidade de mobilização da estrutura (Tabela 2). É importante mencionar que esses indicadores não mostram efetividade para lidar com surtos de vírus corona, mas a capacidade de mobilização. Não obstante, Niterói e Vassouras mantêm as Unidades de Terapia Intensiva, em especial para pacientes afetados pela COVID-19, com o apoio do governo do estado, assim como ocorre na capital. É interessante observar que o município de Volta Redonda, contemplado também pelo sistema estadual, não ocupa posição de destaque. 
RAP | Capacidade do sistema de saúde nos municípios do Rio de Janeiro: infraestrutura para enfrentar a COVID-19

TABELA 2 EVOLUÇÃO DO IEE NAS CIDADES DE MELHOR CLASSIFICAÇÃO

\begin{tabular}{|c|c|c|c|c|}
\hline \multirow[b]{2}{*}{ Grupo } & \multirow[b]{2}{*}{ Cidades } & \multicolumn{2}{|c|}{ I.E.E. } & \multirow[b]{2}{*}{ Acréscimo ou Decréscimo } \\
\hline & & 2016 & 2020 & \\
\hline \multirow[t]{13}{*}{1} & Vassouras & 0,766 & 0,720 & $-0,046$ \\
\hline & Quissamã & 0,400 & 0,500 & 0,100 \\
\hline & Niterói & 0,510 & 0,458 & $-0,052$ \\
\hline & Porto Real & 0,434 & 0,436 & 0,002 \\
\hline & Rio Claro & 0,253 & 0,380 & 0,127 \\
\hline & Macuco & 0,409 & 0,377 & $-0,031$ \\
\hline & Petrópolis & 0,318 & 0,370 & 0,052 \\
\hline & Armação dos Búzios & 0,321 & 0,370 & 0,049 \\
\hline & Miguel Pereira & 0,256 & 0,365 & 0,109 \\
\hline & São Sebastião do Alto & 0,270 & 0,352 & 0,081 \\
\hline & Angra dos reis & 0,329 & 0,351 & 0,023 \\
\hline & São João da Barra & 0,416 & 0,350 & $-0,065$ \\
\hline & Carmo & 0,346 & 0,344 & $-0,002$ \\
\hline \multirow{14}{*}{2} & Natividade & 0,363 & 0,344 & $-0,019$ \\
\hline & Comendador Levy Gasparian & 0,379 & 0,339 & $-0,040$ \\
\hline & Itaperuna & 0,450 & 0,336 & $-0,113$ \\
\hline & Macaé & 0,297 & 0,336 & 0,039 \\
\hline & Cardoso Moreira & 0,222 & 0,323 & 0,101 \\
\hline & Campos dos Goytacazes & 0,325 & 0,320 & $-0,004$ \\
\hline & Sumidouro & 0,128 & 0,319 & 0,191 \\
\hline & Três Rios & 0,368 & 0,312 & $-0,056$ \\
\hline & Carapebus & 0,366 & 0,312 & $-0,054$ \\
\hline & Areal & 0,240 & 0,306 & 0,066 \\
\hline & Valença & 0,248 & 0,305 & 0,057 \\
\hline & Rio das Flores & 0,260 & 0,305 & 0,045 \\
\hline & Rio de Janeiro & 0,315 & 0,298 & $-0,017$ \\
\hline & Bom Jesus do Itabapoana & 0,263 & 0,297 & 0,033 \\
\hline \multirow{4}{*}{3} & Paraíba do Sul & 0,277 & 0,296 & 0,019 \\
\hline & Volta Redonda & 0,272 & 0,296 & 0,024 \\
\hline & Laje do Muriaé & 0,278 & 0,294 & 0,016 \\
\hline & Resende & 0,278 & 0,285 & 0,006 \\
\hline
\end{tabular}

REVISTA de AdMinistração Pública | Rio de Janeiro 54(4):578-594, jul. - ago. 2020 


\begin{tabular}{ccccc}
\hline & \multicolumn{3}{c}{ I.E.E. } \\
Grupo & Cidades & 2016 & 2020 & Acréscimo ou Decréscimo \\
\hline Aperibé & 0,240 & 0,281 & 0,041 \\
Cambuci & 0,282 & 0,279 & $-0,003$ \\
Piraí & 0,368 & 0,278 & $-0,090$ \\
Cantagalo & 0,233 & 0,267 & 0,034 \\
Maricá & 0,114 & 0,265 & 0,151 \\
& Rio Bonito & 0,319 & 0,264 & $-0,055$ \\
Nova Friburgo & 0,216 & 0,257 & 0,041 \\
São José de Ubá & 0,383 & 0,255 & $-0,128$ \\
Itatiaia & 0,272 & 0,254 & $-0,018$
\end{tabular}

Fonte: Elaborada pelos autores.

\section{CONCLUSÕES E SUGESTÕES PARA GESTORES}

O mapeamento do IEE em todos os municípios do estado do Rio de Janeiro resultou como uma ferramenta de classificação frutífera. As anotações exibem a capacidade de mobilização da estrutura de saúde, permitindo melhor precisão na tomada de decisões. Especialmente, sobre a necessidade de um foco regional para resolver problemas de infraestrutura de sistemas de saúde.

Quando comparamos indicadores entre os anos de 2016 e 2020, conseguimos verificar cidades com destaques positivos e negativos, porém, houve uma baixa mobilidade entre os grupos estabelecidos. O fato pode ser bem explicado, uma vez que a busca pela expansão da estrutura, substanciada por hospitais de campo e compra respiradores, foi regulada pela União, com esforços de vários municípios. Como o IEE é um indicador de classificação dentro da própria amostra, formado por seis tipos de dados diferentes, notamos uma limitação de mobilização, ou seja, a dificuldade de um município subir ou descer em muitas posições do ranking.

Considerando os esforços municipais e estaduais para abertura de leitos, não podemos esquecer de apontar a necessidade de mapear leitos existentes e com possibilidade de reativação. Como se não bastasse o cenário de escassez de recursos e investimentos, existem vestígios de possíveis compras irregulares no setor de saúde, o que pode aprofundar os problemas de infraestrutura.

Até o momento da elaboração do presente relatório de pesquisa, as medidas de distanciamento social no Estado estiveram na faixa de 0,3 e 0,6, de acordo com os padrões de Walker et al. (2020), e a contaminação pode atingir mais de cem mil pessoas rapidamente no Estado e cerca de 10.000 mortes, em menos de seis meses após a confirmação do primeiro caso. $\mathrm{O}$ cálculo do distanciamento social não está considerando a desobediência aos decretos atuais. Ele considera a capacidade atual do sistema (o número pode ser mitigado, com a expansão imediata da capacidade, uma ação já iniciada pelo Governo do Estado). Nesse sentido, não é indicado o afrouxamento das medidas, sem o devido cuidado, o que pode dobrar o número de óbitos.

O mais apropriado seria intensificar as medidas de mitigação e adquirir os testes COVID-19 para começar a agir na supressão. Isso significa a necessidade de testar os casos suspeitos mais rapidamente 
RAP | Capacidade do sistema de saúde nos municípios do Rio de Janeiro: infraestrutura para enfrentar a COVID-19

e, consequentemente, isolar os casos confirmados, com a possibilidade de testar os grupos sociais em contato com todos os casos contaminados confirmados. Além disso, o Governo do Estado deve procurar romper o caminho da transmissão do vírus, mantendo a imposição de distanciamento social. Mesmo as necessidades econômicas de curto prazo não podem se sobrepor ao problema. Evidências apontadas por Correia, Luck, e Verner (2020) demonstram que durante a pandemia de gripe de 1918, alguns locais optaram por uma política mais branda de isolamento e outros menos. As áreas com menor distanciamento social apresentaram taxas de doença persistentemente mais altas e piores resultados econômicos. Ao mesmo tempo, as cidades que agiram mais cedo e mais agressivamente começaram a crescer mais rapidamente após o fim da pandemia. 


\section{REFERÊNCIAS}

Baldwin, R., \& Di-Mauro, B. W. (2020). Introduction. In: R. Baldwin; \& B. W. Di-Mauro (Eds.), Mitigating the COVID Economic Crisis: Act Fast and Do Whatever It Takes. London, UK: CEPR Press.

Brindle, M. E., \& Gawande, A. (2020, 21 de maio). Managing COVID-19 in Surgical Systems. Annals of Surgery. Recuperado de https://doi.org/10.1097/ SLA.0000000000003923

Cadastro Nacional de Estabelecimentos de Saúde (2020). Data base of beds. Brasília, DF: Datasus.

Chen, S., Zhang, Z., Yang, J., Wang, J., Zhai, X., Bärnighausen, T., ... Wang, C. (2020). Fangcang shelter hospitals: a novel concept for responding to public health emergencies. The Lancet, 395, 1305-1314.

Correia, S., Luck, S., \& Verner, E. (2020, 30 de março). Pandemics Depress the Economy, Public Health Interventions Do Not: Evidence from the 1918 Flu. Recuperado de https://ssrn.com/abstract $=3561560$

Coutinho, D. C. M., \& Dos-Santos, R. (2019). Política De Saúde No Brasil Pós Constituição Federal de 1988: Reflexões Sobre a Trajetória do SUS. Humanidades \& Inovação, 6(17), 112-126.

Decree 47.025 (2020, 07 de abril). Provides for the release of commercial activity in municipalities without notification of the commitment of COVID-19. Official Gazette of the State Government of Rio de Janeiro.

Farias, L. O., \& Melamed, C. (2003). Segmentação de mercados da assistência à saúde no Brasil. Ciência \& Saúde Coletiva, 8(2), 585-598.

Gilbert, M., Pullano, G. Pinotti, F., Valdano, E., Poletto, C., Böelle, P. Y., ... Colizza, V. (2020). Preparedness and vulnerability of African countries against importations of COVID-19: a modelling study. The Lancet, 395, 871-877.

Gourinchas, P. O. (2020). Flattening the Pandemic and Recession Curves. In: R. Baldwin, B. W. DiMauro (Eds.), Mitigating the COVID Economic Crisis: Act Fast and Do Whatever It Takes. London, UK: CEPR Press.

Keesara, S., Jonas, A., \& Schulman K. (2020, 04 de junho). Covid-19 and health care's digital revolution. The New England Journal of Medicine, 382(23), e82(1)-e82(3). Recuperado de https://doi. org/10.1056/NEJMp2005835
Linschoten, M., \& Asselbergs, F.W. (2020, 14 de maio). CAPACITY-COVID: a European Registry to determine the role of cardiovascular disease in the COVID-19 pandemic. European Heart Journal, 41(19), 1795-1796. Recuperado de https://doi. org/10.1093/eurheartj/ehaa280

Maves, R. C., Downar, J., Dichter, J. R. ... Christian, M. D. (2020, 23 de abril). Triage of scarce critical care resources in COVID-19: an implementation guide for regional allocation: an expert panel report of the Task Force for Mass Critical Care and the American College of Chest Physicians. Chest, Special Features (article in press), 1-14. Recuperado de https://doi. org/10.1016/j.chest.2020.03.063

Mendes, A. N. (2005). Financiamento, gastos e gestão do Sistema Único de Saúde: a gestão descentralizada semiplena e plena do Sistema Municipal no Estado de São Paulo (1995-2001) (Tese de Doutorado). Instituto de Economia da Unicamp, Campinas, SP, Brasil.

Paneru, H. R. (2020). Intensive care units in the context of COVID-19 in Nepal: current status and need of the hour. Journal of Society of Anesthesiologists of Nepal, 7(1), e291-e291.

Rizzotto, M.L.F. (2018) A reafirmação da democracia e do direito universal à saúde em tempos de ultraneoliberalismo. Ciênciae Saúde Coletiva, 23(6), 1-2.

Souza, M. F., Malta, D. C.; Franca, E. \& Barreto, M. (2018). Transição da saúde e da doença no Brasil e nas Unidades Federadas durante os 30 anos do Sistema Único de Saúde. Ciênc. saúde coletiva, 23(6), 1737-1750. Recuperado de https://doi. org/10.1590/1413-81232018236.04822018

Shigemura, J., Ursano, R. J., Morganstein, J. C., Kurosawa, M., \& Benedek, D. M. (2020). Public responses to the novel 2019 coronavirus (2019$\mathrm{nCoV}$ ) in Japan: Mental health consequences and target populations. Psychiatry and clinical neurosciences, 74(4), 281-282. Recuperado de https:// doi.org/10.1111/pcn.12988.

Walter, P. G. T., Whittaker, C., Watson, O., Baguelin, M., Ainslie, K. E. C., Bhatia, S. ... \& Ghani, A. C. (2020). The Global Impact of COVID-19 and Strategies for Mitigation and Suppression. London, UK: Imperial College.

Zheng, M. H., Boni, L., \& Fingerhut, A. (2020, 26 de março). Minimally Invasive Surgery and the Novel Coronavirus Outbreak: Lessons Learned in China and Italy. Annals of surgery. Recuperado de https:// doi.org/10.1097/SLA.0000000000003924 


\section{Giuliano Alves Borges e Silva}

https://orcid.org/0000-0003-4842-7348

Doutor em Administração de Empresas pela Universidade de São Paulo (USP); Professor adjunto do Departamento de Administração de Macaé da Universidade Federal Fluminense (UFF) e do Programa de Pós-Graduação em Administração (PPGAd UFF); Coordenador do Grupo Interdisciplinar de Pesquisa em Gestão, Práticas e Políticas Públicas (GIPP / UFF). E-mail: giulianoalves@id.uff.br

\section{Ernani Viana Saraiva}

https://orcid.org/0000-0001-7548-6330

Doutor em Administração pela Universidade Federal de Minas Gerais; Professor Adjunto da Universidade Federal Fluminense do Departamento de Administração de Macaé; Membro do Grupo Interdisciplinar de Pesquisa em Gestão, Práticas e Políticas Públicas (GIPP/UFF). E-mail: ernanisaraiva@id.uff.br

\section{Guilherme José Santos Nunes Ferreira}

https://orcid.org/0000-0003-2005-1385

Bacharel em Administração; Membro do Grupo Interdisciplinar de Pesquisa em Gestão, Práticas e Políticas Públicas (GIPP/UFF). E-mail: guilherme_jose@id.uff.br

\section{Rogério de Mesquita Peixoto Junior}

https://orcid.org/0000-0002-9459-2988

Graduado em Administração de Empresas pela Universidade Federal Fluminense; Membro do Grupo Interdisciplinar de Pesquisa em Gestão, Práticas e Políticas Públicas (GIPP/UFF).

E-mail: rogeriopeixoto@id.uff.br

\section{Luiz Felipe Ferreira}

https://orcid.org/0000-0002-2045-6107

Graduado em Administração de Empresas pela Universidade Federal Fluminense; Membro do Grupo Interdisciplinar de Pesquisa em Gestão, Práticas e Políticas Públicas (GIPP/UFF).

E-mail: luizfelipeferreira@id.uff.br 